\title{
Catalytic Hydroxyalkylation/Alkylation of 2-Methylfuran with 2 Butanal to Form a Biodiesel Precursor Using Acidic lon-Exchange ${ }_{3}$ Resins
}

${ }_{4}$ Eliana Ramírez,* Rodrigo Soto, Roger Bringué, Montserrat Iborra, and Javier Tejero

Cite This: https://dx.doi.org/10.1021/acs.iecr.0c04308

Read Online

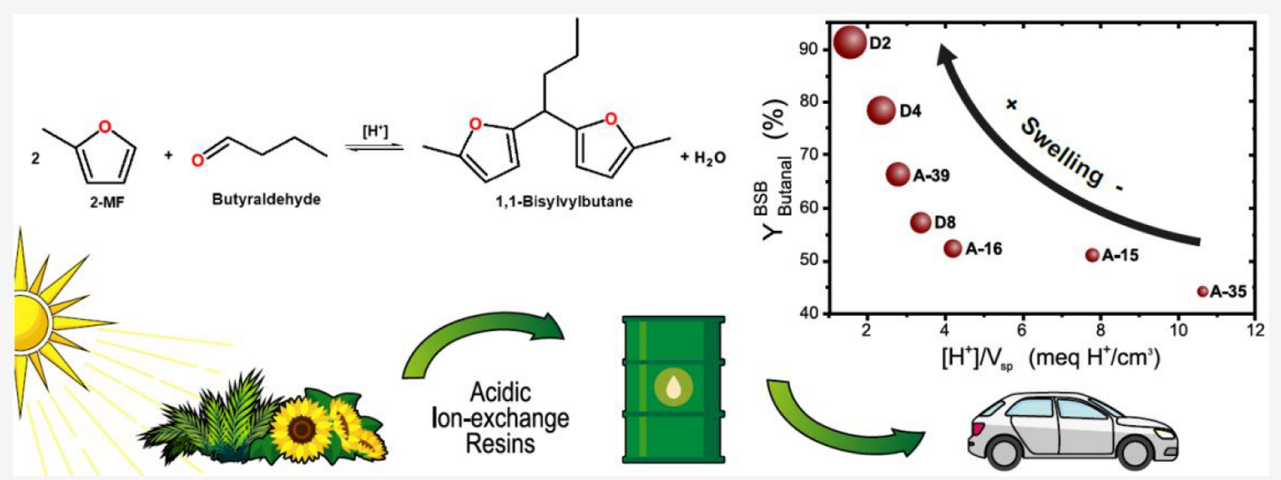

5 ABSTRACT: The catalytic hydroxyalkylation/alkylation of 2-methylfuran (2MF) with butanal has been investigated over several 6 acidic ion-exchange resins within the temperature range $50-90{ }^{\circ} \mathrm{C}$ and at a stoichiometric reactant molar ratio of $2 \mathrm{MF} / \mathrm{butanal}$ 7 (2:1). Butanal conversion increases with temperature and also the formation of undesired 2-methylfuran oligomers, leading to a 8 decrease in yield of the target product. The highest butanal conversion (90\%) is achieved at $50{ }^{\circ} \mathrm{C}$ over Dowex $50 \mathrm{Wx} 2$ with a 9 negligible formation of 2-methylfuran oligomers. The observed catalytic activity and final yield of the target product have been 10 rationalized on the basis of morphological properties of resins and their dynamic behavior within the present reaction medium. The 11 findings reveal that gel-type resins are more active and render higher product yields than their macroreticular congeners due to the 12 enhanced accessibility to acid centers because of their improved ability to swell throughout the reaction. Macroreticular resins with a 13 low cross-linking degree, e.g., Amberlyst39, also produce interesting catalytic results. The stability of the most promising catalyst has 14 been evaluated after three reaction cycles, and the full reusability outcome speaks for its appropriateness as a potential catalyst for the 15 studied process.

\section{INTRODUCTION}

16 The continuous exploitation of irreplaceable oil reserves and 17 the ensuing increase of its derived environmental effects has 18 generated the need for green alternative fuels, platforms, and 19 fine chemicals. From this scenario, biomass emerges as the 20 only renewable, widespread, abundant, and cheap source of 21 carbon-based materials that can be considered a plausible 22 substitute for petroleum. ${ }^{1}$ The hydrolysis of biomass is a 23 selective process of depolymerization by dissociation of energy24 neutral $\mathrm{C}-\mathrm{O}$ bonds that yields $\mathrm{C} 5$ and $\mathrm{C} 6$ monosaccharides, 25 e.g., glucose, fructose, and xylose, which preserve the energy26 profitable $\mathrm{C}-\mathrm{H}$ and $\mathrm{C}-\mathrm{C}$ bonds. ${ }^{2,3}$ Further processing of 27 these hydrocarbons renders a wide array of platform chemicals, 28 e.g., levulinic acid, ${ }^{4,5}$ 5-hydroxymethylfurfural, furfural, and 229 methylfuran, ${ }^{6}$ which can be used for the synthesis of highly 30 valuable components for transportation fuels and the 31 production of fine chemicals. ${ }^{7}$ Among them, 2-methylfuran
$(2 \mathrm{MF} \text {, also known as sylvan })^{8}$ obtained from furfural has 32 recently attracted interests for biofuel production due to its 33 versatility to direct blending with gasoline ${ }^{9}$ and diese ${ }^{10}$ and to 34 synthesize high-density biofuel. ${ }^{11}$ For instance, the hydrox- 35 yalkylation/alkylation (HAA) of sylvan with $n$-butanal 36 produces 1,1-bisylvylbutane (BSB), which can be transformed 37 into 6-propyl undecane by a subsequent hydrodeoxygenation 38 (HDO) step in series. Using platinum-supported carbon- or 39 alumina-based catalysts, a mixture of C9, C12, and C14 alkanes 40 can be obtained as an organic phase from the second reaction 41

Received: September 1, 2020

Revised: October 21, 2020

Accepted: November 4, 2020 
Scheme 1. Hydroxyalkylation/Alkylation of Sylvan with Butyraldehyde to Produce 1,1-Bisylvylbutane (BSB)

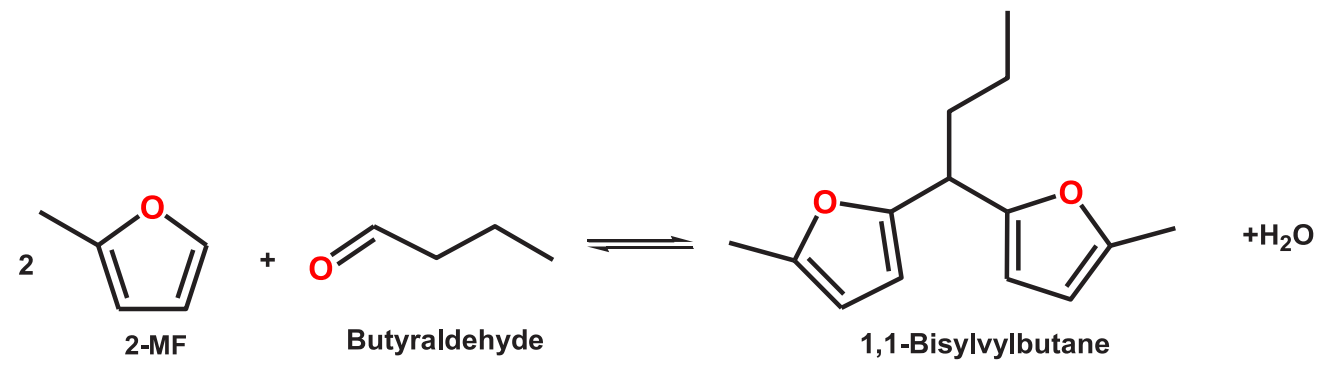

Scheme 2. Self-Condensation of 2-Methylfuran to Form Its Trimer and Tetramers

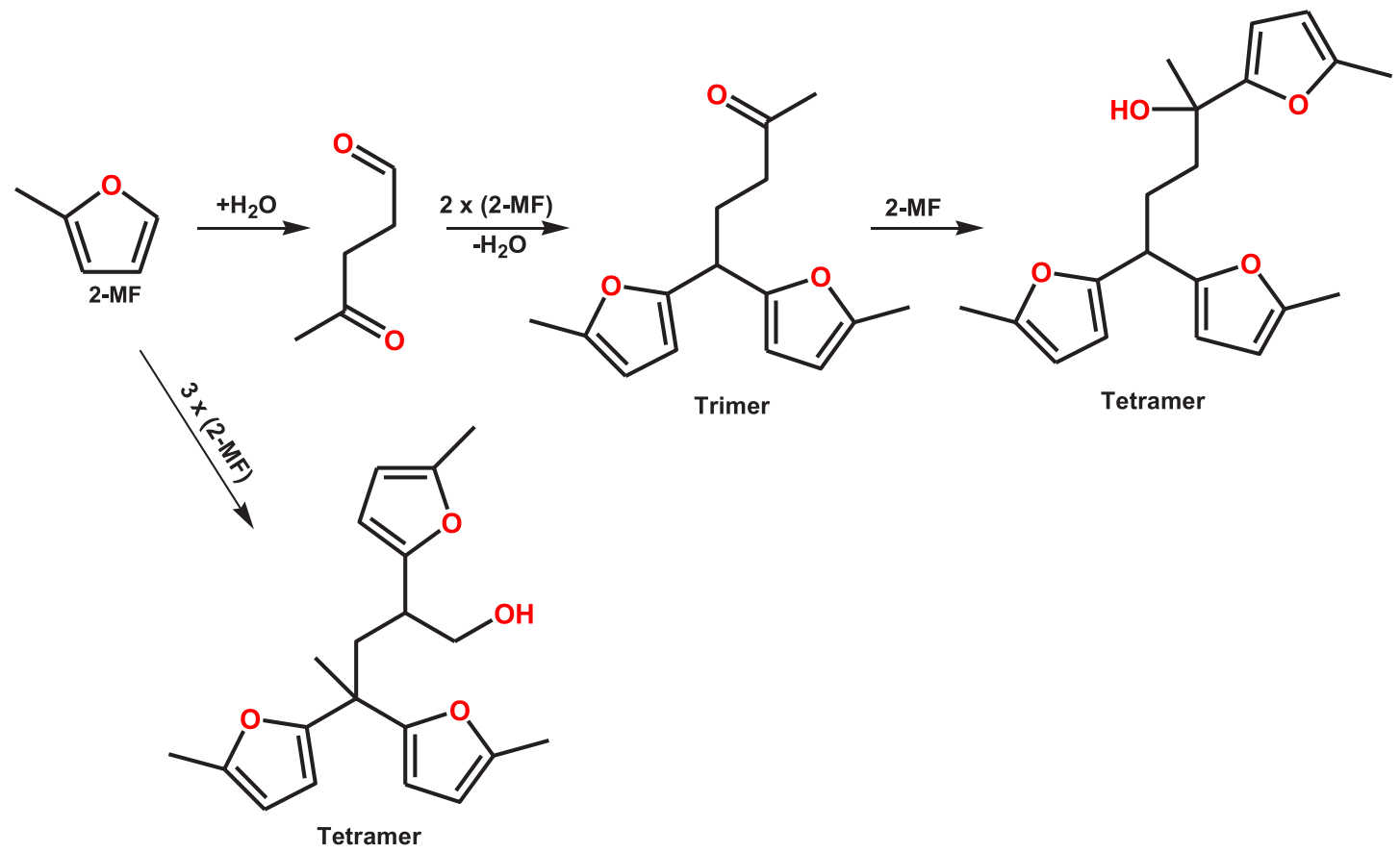

42 step $^{12}$ with excellent diesel fuel properties, e.g., pour point of $43-90{ }^{\circ} \mathrm{C}$ and cetane number of $71 . .^{13}$ In the first reaction step, 44 sylvan molecules can react with different aldehydes and 45 ketones to give a precursor with an adequate carbon atom 46 number that can be further hydrogenated to yield $\mathrm{C}_{12}{ }^{+}$ 47 oxygenated intermediate molecules. Such a pathway is 48 illustrated in Scheme 1 using butanal as an alkylating agent. 49 The use of butanal presents the advantage of being a 50 bioreactant since it can be produced by partial oxidation or 51 dehydrogenation of biobutanol obtained from the acetone52 butanol-ethanol (ABE) fermentation process. ${ }^{14}$

53 During the cross-condensation of 2-methylfuran with $n$ 54 butanal to form BSB (Scheme 1), some side reactions can also 55 take place (Scheme 2) originated from the self-condensation of $562 \mathrm{MF}$ that produces a trimer (5,5-bis (5-methylfuran-2-yl) 57 pentan-2-one or 5,5-bisylvyl-2-pentanone) and a tetramer 58 (2,4,4-trisylvyl-2-pentanol or 2,4,4-tris(5-methylfuran-2-yl)59 pentan-2-ol). $8,14,15$ An implicit drawback that makes working 60 with $2 \mathrm{MF}$ challenging is its ease to polymerize in the presence 61 of acid catalysts, even under moderate acid conditions, e.g., 62 phosphoric acid. ${ }^{16}$ Accordingly, different types of catalysts have 63 been tested in the hydroxyalkylation/alkylation of sylvan with 64 carbonyl compounds, ${ }^{3,8,17}$ aiming to control polymerization 65 that results in a brown viscous liquid comprising tetra-, penta-, 66 hexa-, and heptamers.
Interesting yields and conversions ranging from 60 to $100 \% 67$ have been reported for the HAA of sylvan with butanal 68 (Scheme 1) over different types of catalysts such as 69 $\mathrm{NbOPO}_{4}{ }^{18}{ }^{18}$ improved graphene oxide, ${ }^{19}$ copper(II) triflate, ${ }^{20} 70$ Sn-beta(12.5) zeolite, $^{21}$ Nafion 212, ${ }^{15,22}$ Amberlyst15 71 (A15), ${ }^{8,12,15,22}$ and Amberlyst36 (A36), ${ }^{15,22}$ Dowex 72 $50 \mathrm{~W} 2,{ }^{8,12}$ protonated titanate nanotubes, ${ }^{23} \mathrm{KCC}-73$ $1 \mathrm{APSO}_{3} \mathrm{H}^{15}$ and acidic carbon catalysts 74 $\left(60 \mathrm{LS} 40 \mathrm{PS} 350 \mathrm{H}^{+}\right) .^{24}$ Further details on the experimental 75 conditions and results reported are provided in section 3.176 for a proper comparison.

The state of the art reveals that the range of reaction 78 temperatures for the HAA of sylvan is fully compatible with the 79 operating temperatures of standard ion-exchange resins $(<15080$ ${ }^{\circ} \mathrm{C}$ ), whose active sites can catalyze the reaction in Scheme $1 .{ }^{25} 81$ However, the number of studies for this reaction over acidic 82 ion-exchange resins is scarce and limited to only a few resins, 83 being Amberlyst (A15) the most frequently used. Interestingly, 84 A15 is of macroreticular nature and, hence, of questionable 85 application for systems in which water is a reaction product 86 and that involve products of relatively big molecular volume, 87 for which gel-type resins should be more suitable. ${ }^{26}$ Ion- 88 exchange resins are environmentally friendly catalysts because 89 of their nontoxicity, noncorrosiveness, and cost-effective 90 recyclability. They can be synthesized in a wide variety of 91 
92 tunable properties, e.g., acid capacity, pore diameter, and cross93 linking degree, making them excellent catalysts to investigate 94 the relations between catalytic activity and catalysts morphol95 ogy for different applications.

96 The present work aims to shed light in this regard by the 97 evaluation of several ion-exchange resins of different character98 istics for the HAA of sylvan with butanal. The main objective is 99 to identify the optimum catalyst properties and experimental 100 conditions that maximize BSB production and minimize the 101 extent of side reactions. Therefore, special emphasis is devoted 102 to a comprehensive analysis that allows for rationalizing the 103 relations between catalysts' morphological properties and the 104 reported catalytic activity. In addition, the stability of the best 105 catalyst selected is evaluated by several reaction cycles in the 106 most promising conditions.

\section{EXPERIMENTAL SECTION}

107 2.1. Chemicals. Butyraldehyde (butanal, 99\% dry) and 2108 methylfuran (sylvan, 2MF, 99\%), both supplied by Sigma109 Aldrich, were used as reactants and standards without further 110 purification. As BSB is not a commercial product, its standard 111 for calibration was obtained from the organic phase of 112 preliminary experiments. After distillation, the purity of BSB 113 was $98 \%$ GC. Nitrogen (99.999\% GC) and helium (99.998\% 114 GC) supplied by Air Liquid were used to pressurize the system 115 and for the chromatographic analyses. For catalyst reusability 116 tests, dry methanol (0.005 wt \% of water, Panreac 117 AppliChem), water (Milli-Q, Millipore Corp.), and hydrogen 118 peroxide $(30 \% \mathrm{w} / \mathrm{v}$, Panreac AppliChem) were used as 119 reagents.

120 2.2. Catalysts. A series of gel-type and macroreticular 121 acidic polystyrene-divinylbenzene (PS-DVB)-based ion-ex122 change resins were used as catalysts to check their performance 123 in hydroxyalkylation/alkylation of 2-methylfuran with butanal. 124 These were Amberlyst15 (A15), Amberlyst16 (A16), Amber125 lyst35 (A35), Amberlyst39 (A39), Dowex 50Wx2 (D2), 126 Dowex 50Wx4 (D4), and Dowex 50Wx8 (D8). All resins 127 were supplied in wet form and used in the as-received particle 128 size. The mesh size distribution of Dowex resins was 50-100. 129 Resins were first dried and activated for $2 \mathrm{~h}$ at $120^{\circ} \mathrm{C}$ at $1 \mathrm{~atm}$ 130 and then overnight under a vacuum at $100{ }^{\circ} \mathrm{C}$ and $0.1 \mathrm{mbar}$. 131 The final water content after drying was 3-4 wt \% (Karl 132 Fischer titration).

133 2.3. Apparatus and Analytical Methods. The exper134 imental setup consisted of a $200 \mathrm{~mL}$ stainless steel stirred tank 135 batch reactor equipped with a six-blade magnetic stirrer 136 (Autoclave Engineers; PA, USA). The working temperature 137 range was $50-90{ }^{\circ} \mathrm{C}$, controlled within $\pm 0.1{ }^{\circ} \mathrm{C}$ by means of a 138 thermostatic bath filled with a 50 vol \% mixture of propylene 139 glycol and water. The reactor pressure was maintained at 1.5 $140 \mathrm{MPa}$ with nitrogen to widely exceed the vapor pressure of the 141 reaction mixture at the highest assayed temperature to ensure 142 that the reaction was performed in the liquid phase and to 143 impel samples from the reactor to the gas chromatograph.

144 The reaction mixture was analyzed by injecting online 145 samples of $0.2 \mu \mathrm{L}$ of pressurized liquid in a gas chromatograph 146 (Agilent 6890, US) equipped with a capillary column HP 147 PONA 19091S-001 (5\% phenyl methyl siloxane $50.0 \mathrm{~m} \times 0.2$ $148 \mathrm{~mm} \times 0.5 \mu \mathrm{m}$ nominal) and a mass spectrometer detector 149 (Agilent 5973N, US). A second gas chromatograph (Hewlett150 Packard GC 6890A), equipped with a TCD detector, was used 151 to measure the water content by injecting liquid samples of 0.2 $152 \mu \mathrm{L}$, taken from the reactor after finishing the experiment, and using a $50 \mathrm{~m} \times 0.2 \mathrm{~mm} \times 0.5 \mu \mathrm{m}$ methyl silicone HP 90915- 153 001 capillary column. The temperature program consisted of a 154 6 min initial hold at $45{ }^{\circ} \mathrm{C}$, followed by a $30^{\circ} \mathrm{C} \mathrm{m^{-1 }}$ ramp up 155 to $180{ }^{\circ} \mathrm{C}$, held for $5 \mathrm{~min}$. A total flow rate of $30 \mathrm{~mL} \mathrm{~min}^{-1}$ of a 156 carrier gas $(\mathrm{He})$ was used.

157

2.4. Procedure and Calculations. In the screening, a 158 molar stoichiometric ratio $R_{0}(2 \mathrm{MF} /$ butanal $)=2$ was used, 159 corresponding to a $53.28 \mathrm{~g}$ of butanal and $121.54 \mathrm{~g}$ of sylvan. A 160 catalyst load of $1 \mathrm{wt} \%$ was introduced by a pressure drop when 161 the working temperature of $50{ }^{\circ} \mathrm{C}$ had been reached. After 162 that, the reactor was pressurized to $1.5 \mathrm{MPa}$; that moment was 163 considered as the starting reaction time. The typical total 164 duration of the experiments was $5.4 \mathrm{~h}$. A stirring rate of 750165 rpm was considered high enough to avoid the possible 166 influence of external mass transfer. However, some effect of the 167 internal mass transfer is expected since resins were used in 168 commercial particle sizes. This fact has no transcendence in the 169 reported results, as the main screening goal was to compare the 170 catalytic behavior of a series of resins from an industrial 171 application standpoint rather than rigorous kinetic modeling. 172 The effect of the catalyst load ( 1 and 2 wt \%) was also studied 173 by two additional experiments, $8 \mathrm{~h}$ in length, under identical 174 experimental conditions. The effect of the reaction temper- 175 ature was studied within $50-90{ }^{\circ} \mathrm{C}$ using $1 \mathrm{wt} \%$ of the catalyst 176 load. In addition, the resin stability during three reaction cycles 177 was checked for the most promising catalyst. After a typical 178 run, the used resin was filtered and stirred overnight in $50 \mathrm{~mL} 179$ of $30 \mathrm{vol} \% \mathrm{H}_{2} \mathrm{O}_{2}$ for its regeneration. After another filtration, 180 the catalyst was washed with deionized water $(30 \mathrm{~mL} \times 3)$ and 181 methanol $(30 \mathrm{~mL} \times 1)$. Finally, the recovered resin was dried 182 overnight at $100{ }^{\circ} \mathrm{C}$ before reuse.

For each experiment, butanal conversion and yield to BSB 184 were calculated by eqs 1 and 2, respectively. As no butanal- 185 derived byproducts were detected, the butanal selectivity to 186 BSB was always $100 \%$ (at $50{ }^{\circ} \mathrm{C}$ ), and thus, the butanal 187 conversion and yield of BSB were equivalent.

$$
X_{\text {butanal }}=\frac{\{\text { mole of reacted butanal }\}}{\{\text { initial mole of butanal }\}}=\frac{n_{\text {butanal }}^{0}-n_{\text {butanal }}}{n_{\text {butanal }}^{0}}
$$

$$
\begin{aligned}
& Y_{\text {butanal }}^{\mathrm{BSB}}=X_{\text {butanal }} S_{\text {butanal }}^{\mathrm{BSB}} \\
& \quad=\frac{\{\text { mole of reacted butanal to BSB }\}}{\{\text { initial mole of butanal }\}}=\frac{n_{\mathrm{BSB}}}{n_{\text {butanal }}^{0}}
\end{aligned}
$$

To account for the slight variations in the weighed catalyst 191 mass $\left(W_{\text {cat }}\right)$, the reaction time was standardized in terms of 192 contact time (eq 3) for a suitable comparison among catalysts. 193

$$
\text { contact time }=\frac{t W_{\text {cat }}}{n_{\text {butanal }}^{0}}
$$

After evaluating different mathematical expressions to be 195 used as an empirical model, a reciprocal quadratic function of 196 the form $y=x /\left(a+b x+c x^{2}\right)$ was used to fit to the BSB mole 197 evolutions. Subsequently, the formation rates $\left(r_{\mathrm{BSB}}\right)$ at any 198 time $(t)$ were estimated as

$$
r_{\mathrm{BSB}}=\frac{1}{W_{\text {cat }}}\left[\frac{\mathrm{d} n_{\mathrm{BSB}}}{\mathrm{d} t}\right]_{t}
$$

where $W_{\text {cat }}$ is the dry catalyst mass and $n_{\mathrm{BSB}}$ is the BSB mole. 201 Initial reaction rates $\left(r_{\mathrm{BSB}}^{0}\right)$ were calculated at the initial 202 instant, and the catalysts turnover frequencies (TOF) $\left[\mathrm{mol} \mathrm{h}^{-1} 203\right.$ 
$204 \mathrm{eq}^{-1}$ ] were estimated as the quotient of $r_{\mathrm{BSB}}$ to the 205 corresponding acid capacity at the instant considered. For all 206 screening experiments, the mass balance was fulfilled within $207 \pm 5 \%$ on a mole basis.

\section{RESULTS AND DISCUSSION}

208 3.1. Catalyst Load and Screening Studies. Figure 1 209 presents the normalized reactants and BSB mole evolution for

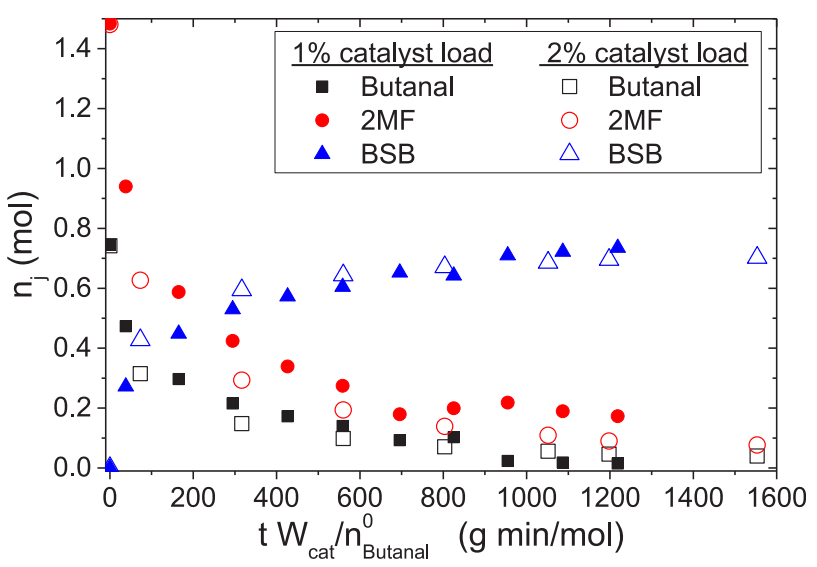

Figure 1. Effect of catalyst load (D2). $T=50{ }^{\circ} \mathrm{C}, 1.5 \mathrm{MPa}, 750 \mathrm{rpm}$, $R_{0}(2 \mathrm{MF} /$ butanal $)=2, t=8 \mathrm{~h}$. Hollow symbols refer to a $2 \% \mathrm{wt}$ catalyst load and filled ones to a $1 \%$ wt.

210 the different catalyst loads evaluated using Dowex 50Wx2 211 (D2). As expected, the consumption of the reactants was 212 accompanied by an increase of product formed with a 213 consistent fulfillment of the mass balance. Within the 214 experimental error, the molar ratio between reactants was 215 maintained during their consumption throughout the experi216 ment. Noteworthy, the virtually steady composition after $6 \mathrm{~h}$ 217 was near chemical equilibrium, suggesting a high reaction 218 equilibrium constant because the BSB formed mole coincides 219 with the initially loaded butanal, in agreement with the 220 reaction stoichiometry. The acceptable overlapping of the 221 corresponding series reveals a negligible effect of the catalyst 222 load. Hereinafter, a loading of 1 wt \% will be used for the rest 223 of the runs.

224 Bearing in mind the physicochemical nature of resins is 225 essential for understanding their catalytic behavior and for a 226 rational interpretation of results. Acidic resins are styrene227 divinylbenzene copolymers, in which sulfonic groups are linked 228 to the benzene ring of styrene as active sites. In a 229 conventionally sulfonated resin, the structure holds a 230 maximum of one sulfonic group per styrene ring, ${ }^{27}$ whereas, 231 for an oversulfonated resin, such proportion is higher than 232 unity. $^{28}$ Gel-type resins are typically translucent beads of 233 homogeneous microstructures without discontinuities. The 234 matrix of such kind of resins is obtained by polymerization in 235 the absence of a solvent called "porogen", and therefore, they 236 do not have permanent pores in a dry state. In this impervious 237 structure, the polymeric matrix is collapsed and renders an 238 almost inactive catalyst after sulfonation because only a few 239 acid centers over the beads external surface are accessible. 240 Conversely, macroreticular resins are opaque beads obtained in 241 the presence of a porogen whose elimination produces a 242 macroporous structure. The resulting net of pores is 243 permanent regardless of the polymer matrix swelling, even at 244 nil swelling conditions in a dry state. ${ }^{29,30}$ In short, the catalytic activity of resin will depend strongly on the accessibility to 245 active sites, which, in turn, is dependent on the bulk properties 246 of the resin's working environment.

Gel-type resins primarily exhibit catalytic activity in a 248 medium capable of expanding the polymeric matrix, i.e., when 249 the solubility parameter of the medium (typically in polar 250 solvents, reactants, or products) is similar to that of the resins. 251 On the other hand, macroreticular resins exhibit catalytic 252 activity in both swelling and nonswelling conditions due to 253 their permanent porosity. ${ }^{27,31,32}$ As an illustrative representa- 254 tion, Figure 2 shows the three types of pores found in a $255 \mathrm{f} 2$

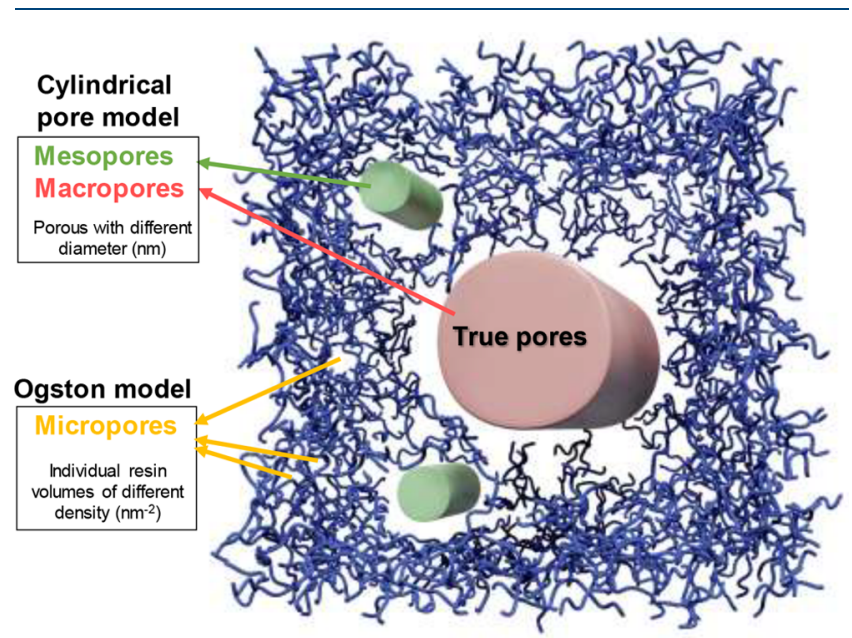

Figure 2. Schematic representation of the types of pores in swollen macroreticular resins and linking to the models applied for their description.

macroreticular resin in a swollen state: (i) micropores of the 256 nonswelling inaccessible part of the polymeric matrix, (ii) new 257 mesopores formed by swelling, and (iii) macropores from the 258 permanent porosity. However, gel-type resins in the swollen 259 state contain only two types of pores (i and ii), which gradually 260 vanish during shrinking. The extension of the microporous and 261 mesoporous zones depends on the swelling degree. In highly 262 polar mediums, such as water, the resin will be very swollen, 263 showing accessibility to practically all active sites. The 264 inaccessible zone of micropores is negligible, and the entire 265 polymer matrix presents a mesoporous structure. Accordingly, 266 the resin's morphological properties are dynamic and depend 267 on the reaction medium polarity, which often varies with the 268 course of the reaction. The understanding of such a complex 269 scenario is paramount to interpret the results comprehensively. 270 For instance, it can be useful to link the catalytic behavior to 271 the resin properties of the resin in a dry state for nonpolar 272 conditions, whereas it is more reasonable to link the behavior 273 of the resin to properties obtained in a swollen state for polar 274 reaction mediums. Consequently, approaches based on 275 porosimetric information (nitrogen adsorption/desorption 276 and mercury porosimetry) are more appropriate to understand 277 resin performance in nonpolar conditions, but they become 278 unsuitable for explaining the behavior under swelling in the 279 presence of polar solvents.

Inverse steric exclusion chromatography (ISEC) ${ }^{33}$ is a useful 281 characterization technique for describing the resin morphology 282 in a swollen state. In the fundamentals of that method, 283 macroporous and mesoporous regions are simulated by the 284 cylindrical pore model, while microporous zones are simulated 285 
Table 1. Characteristics and Structural Parameters of Resins in the Dry State and Swollen in Water

\begin{tabular}{|c|c|c|c|c|c|c|c|c|c|c|}
\hline \multirow[b]{2}{*}{ type ${ }^{a}$} & \multirow[b]{2}{*}{ catalyst } & \multirow[b]{2}{*}{ sulfonation type $\mathrm{e}^{b}$} & \multirow[b]{2}{*}{$T_{\max }\left({ }^{\circ} \mathrm{C}\right)$} & \multirow[b]{2}{*}[\mathrm{H}^{+}]{$^{c}(\mathrm{mmol} / \mathrm{g})$} & \multirow[b]{2}{*}{ DVB (\%) } & \multicolumn{2}{|c|}{ dry state ${ }^{d}$} & \multicolumn{3}{|c|}{ swollen in water ${ }^{g}$} \\
\hline & & & & & & $S_{\mathrm{g}}^{e}\left(\mathrm{~m}^{2} / \mathrm{g}\right)$ & $V_{\mathrm{g}}^{f}\left(\mathrm{~cm}^{3} / \mathrm{g}\right)$ & $\overline{S_{\mathrm{g}}\left(\mathrm{m}^{2} / \mathrm{g}\right)}$ & $V_{\mathrm{sp}}\left(\mathrm{cm}^{3} / \mathrm{g}\right)$ & {$\left[\mathrm{H}^{+}\right] / V_{\mathrm{sp}}\left(\mathrm{mmol} / \mathrm{cm}^{3}\right)$} \\
\hline M & A35 & OS & 150 & 5.32 & 20 & 34 & 0.21 & 199 & 0.50 & 10.6 \\
\hline M & A15 & CS & 120 & 4.81 & 20 & 42 & 0.33 & 192 & 0.62 & 7.8 \\
\hline M & A16 & CS & 120 & 4.80 & 12 & 1.7 & 0.013 & 46 & 1.14 & 4.2 \\
\hline M & A39 & CS & 130 & 4.81 & $7-8$ & 0.09 & $3 \times 10^{-4}$ & 56 & 1.64 & 2.9 \\
\hline G & D8 & CS & 150 & 4.80 & 8 & 0 & 0 & 0 & 1.40 & 3.4 \\
\hline G & D4 & CS & 150 & 4.95 & 4 & 0.011 & 0 & 0 & 1.90 & 2.6 \\
\hline G & $\mathrm{D} 2$ & CS & 150 & 4.98 & 2 & 0 & 0 & 0 & 2.68 & 1.9 \\
\hline
\end{tabular}

${ }^{a}$ Macroreticular (M) and gel (G). ${ }^{b}$ Conventionally sulfonated (CS) and oversulfonated (OS). ${ }^{c}$ Acid capacity. Titration against a standard base. ${ }^{d}$ By adsorption-desorption of $\mathrm{N}_{2}$ at $77 \mathrm{~K}\left(\mathrm{~N}_{2}\right.$ for $S_{\mathrm{g}} \geq 1 \mathrm{~m}^{2} / \mathrm{g}$; Kr for $\left.S_{\mathrm{g}}<1 \mathrm{~m}^{2} / \mathrm{g}\right) .{ }^{e}$ BET method. ${ }^{f}$ Volume of $\mathrm{N}_{2}$ adsorbed at a relative pressure $P / P_{0}$ 0.99. ${ }^{g}$ ISEC (Inverse steric exclusion chromatography) method.

286 by the geometrical model proposed by Ogston, ${ }^{34}$ in which 287 micropores are described as spaces between randomly oriented 288 rigid rods, representing the polymer chains. The main 289 characteristic parameter from ISEC is the specific volume of 290 the swollen polymer, $V_{\mathrm{sp}}$, in $\mathrm{cm}^{3} / \mathrm{g}$, which includes the volume 291 of the free space plus that occupied by the skeleton. Table 1 292 shows the $V_{\mathrm{sp}}$ of the tested resins, textural properties 293 determined in a swollen and dry state, and some other 294 relevant physicochemical properties. $T_{\max }$ is the maximum 295 temperature for thermal stability, $\left[\mathrm{H}^{+}\right]$is the acid capacity, $296 \mathrm{DVB}$ is the percentage of the cross-linking agent, $S_{\mathrm{g}}$ is the 297 specific surface area, $V_{\mathrm{g}}$ is the volume of pores on the dry state, 298 and $\left[\mathrm{H}^{+}\right] / V_{\mathrm{sp}}$ is the acid density of the swollen polymer. As it 299 can be seen, $V_{\text {sp }}$ decreases as DVB increases both for gel-type 300 and macroreticular resins. Low $V_{\mathrm{sp}}$ values imply a high density 301 of polymer matrix in the swollen state and, as a result, poorly 302 accessible spaces even for small molecules. Conversely, high $303 V_{\text {sp }}$ values are associated with a low density of polymer mass 304 and large spaces, which can be accessible for even large 305 molecules.

306 Figure 3 plots the evolution of the butanal conversion for 307 Dowex gel-type resins. At the end of the runs, $X_{\text {butanal }}$ was

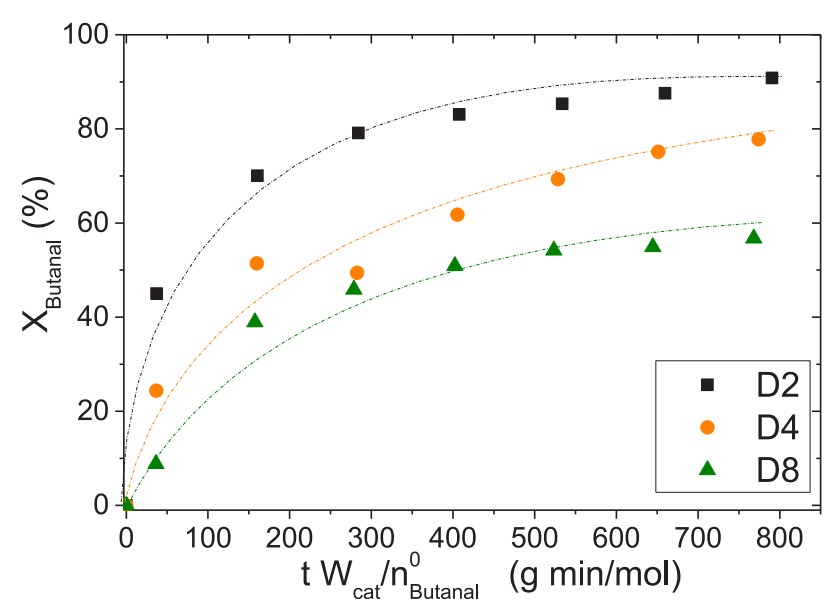

Figure 3. Butanal conversion versus contact time for gel-type resins. $T$ $=50^{\circ} \mathrm{C}, 1.5 \mathrm{MPa}, R_{0}(2 \mathrm{MF} /$ butanal $)=2$, catalyst loading $1 \mathrm{wt} \%, t=$ $5.4 \mathrm{~h}$. Dashed lines are a guide to the eye.

308 about $90 \%$ for the resin D2, which has the lowest cross-linking 309 degree, followed by $80 \%$ for D4 and about $60 \%$ for D8. Among 310 them, D2 was the most active gel-type resin reflected in the 311 steepest curve, indicating a faster reaction rate. As the acid 312 capacity of these resins increases, so does the reported conversions. However, that difference (e.g., between D4 and 313 D2 resins: 4.98 and $4.95 \mathrm{mmol} / \mathrm{g}$ ) does not justify the 314 significantly different activity observed. This suggests that 315 accessibility, and thus swelling, plays a paramount role in the 316 catalytic activity, which can be explained on the basis of the 317 morphology of such type of resins; they progressively swell as 318 the reacting medium becomes more polar, mainly induced by 319 the presence of formed water, enhancing, therefore, accessi- 320 bility to active sites with the course of the reaction. As the 321 resins cross-linking degree decreases (D8 > D4 > D2), the 322 swelling capacity or $V_{\text {sp }}$ increases (see Table 1), leading to an 323 increase in the flexibility of polymer chains and, hence, to 324 improved accessibility to acid sites.

325

Figure 4 shows the butanal conversion standardized $326 \mathrm{f} 4$ evolution for the different macroreticular resins studied. The 327

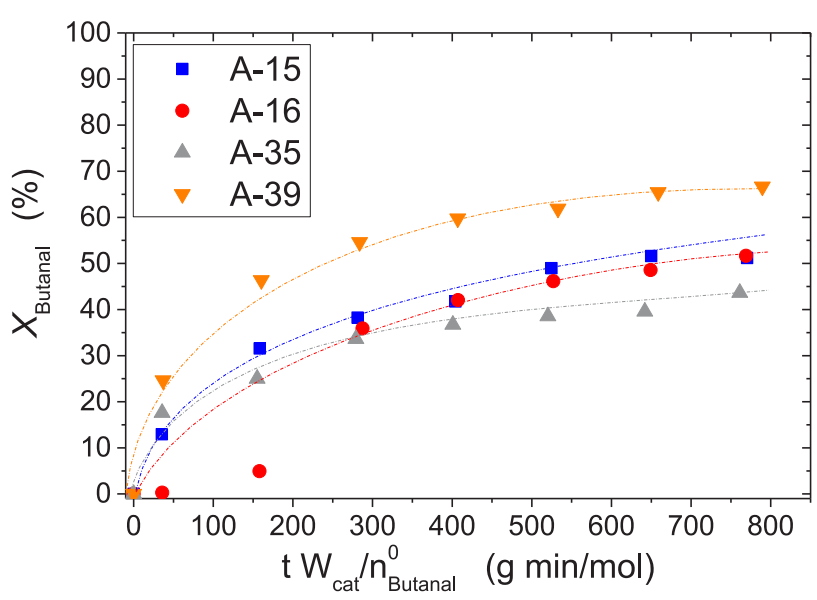

Figure 4. Butanal conversion versus contact time for macroreticular resins. $T=50^{\circ} \mathrm{C}, 1.5 \mathrm{MPa}, R_{0}(2 \mathrm{MF} /$ butanal $)=2$, catalyst loading 1 wt $\%, t=5.4 \mathrm{~h}$. Dashed lines are a guide to the eye.

final achieved conversions follow the order A39 > A15 $\approx \mathrm{A} 16328$ $>$ A35, which again is consistent with the decreasing order of 329 $V_{\text {sp }}$ from Table 1 . This fact clearly shows the significant effect 330 of the resin morphology on catalytic behavior. For the resin 331 A16, the experimental data at about 40 and $160 \mathrm{~g} /(\mathrm{min} \mathrm{mol}) 332$ suggest a sort of induction period. Apart from the inherent 333 experimental uncertainty, this unexpected behavior could be 334 attributed to the lower \% DVB of this resin compared to A15 335 and A35. This results in A16 having a higher ability to swell 336 than A15 and A35; however, its macroreticular and still stiff 337 structure somewhat offers resistance to swelling if compared to 338 D2, D4, and D8 catalysts. Noteworthy, the run using A16 was 339 
340 replicated, and the results confirmed that such behavior was 341 reproducible. As deducted from Figure 3 results, the highest 342 conversion values at the end of the runs were obtained for A39, 343 a resin with a high $V_{\text {sp }}$ and low cross-linking degree, which, as 344 mentioned, encompass lower density of polymer chains, larger 345 flexibility, and wider spaces due to their higher ability to swell. 346 Such type of structure enhances the accessibility of reactants to 347 acid sites as well as the diffusion of big-sized product molecules 348 as BSB from those active sites. This relation applies for both 349 gel-type and macroreticular resins, yet under identical 350 experimental conditions, the activity of macroreticular resins 351 is clearly lower than gel-type resins due to the higher \% DVB. 352 Considering the implicit relationship between the concen353 tration of active sites and catalytic activity, the acid capacity 354 must also be taken into account to assess the final conversion 355 and yield reported values. For this purpose, the final yield 356 toward BSB (target product) can be related to the acid density 357 of swollen polymer $\left[\mathrm{H}^{+}\right] / V_{\mathrm{sp}}$, which is evidently higher for 358 those resins of oversulfonated nature and present a high cross359 linking degree. Figure 5 shows a clear relationship between

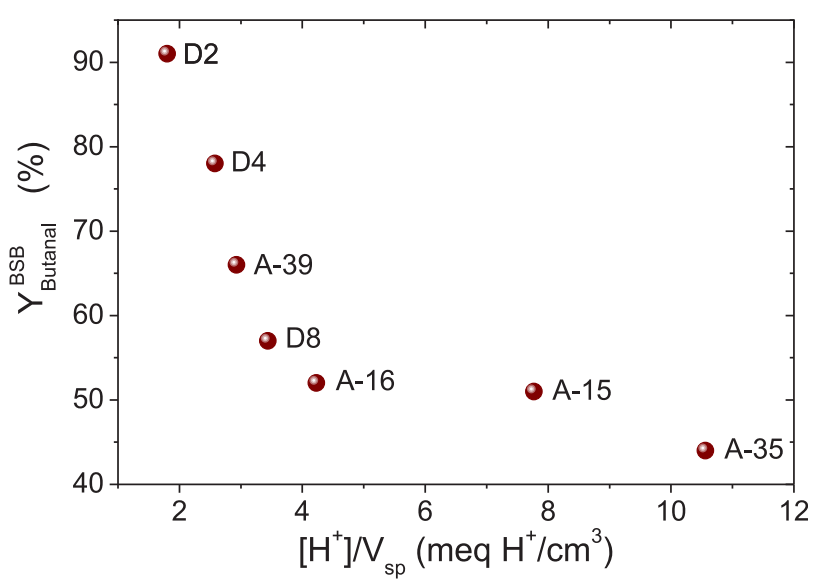

Figure 5. Yield of butanal to BSB, $Y_{\text {butanal, }}^{\mathrm{BSB}}$ versus acid density for macroreticular and gel-type resins. $T=50{ }^{\circ} \mathrm{C}, 1.5 \mathrm{MPa}, R_{0}(2 \mathrm{MF} /$ butanal) $=2$, catalyst loading 1 wt $\%, t=5.4 \mathrm{~h}$.

360 both variables: the final product yield increases with decreasing 361 acid density of the resins. Interestingly, the relation seems to 362 have two clear linear periods: the steepest one including the 363 resins D2, D4, D8, and A39 (with the lowest cross-linking 364 degree) and the less steep period including the macroreticular 365 resins A15, A16, and A35. In comparison with A35, which has 366 the same degree of cross-linking, A15 gives not only a higher 367 butanal conversion and yield but also an apparently faster 368 reaction rate at contact times above $300(\mathrm{~g} \mathrm{~min}) / \mathrm{mol}$ as can be 369 inferred from the steeper slope in Figure 4. This can be 370 explained by the higher pore volume and $V_{\text {sp }}$ of A15 that 371 facilitates the internal diffusion of reactants and formed 372 products.

373 Of course, it is to be noted that resin characterization by 374 ISEC is at swelling conditions in water, and that is not exactly 375 the actual state of the resins in the reaction medium. The initial 376 reaction medium is essentially formed by sylvan and butanal; 377 water was formed as a byproduct with the course of the 378 reaction. Therefore, it could be expected that the resins were 379 not swelled in the initial reaction steps. However, it is to be 380 noted that butanal is also a polar substance, e.g., dipole 381 moment even higher than that of water, and therefore, swelling is expected to occur from the initial steps of the reaction. In 382 this sense, the characterization by ISEC is a reasonable 383 approximation to the morphology of the actual catalysts in 384 reaction conditions, which allows for explaining the catalytic 385 behavior observed for the different catalysts.

386

In order to assess the initial catalytic activity of the resins 387 evaluated, Figure 6 plots the evolution of the turnover $388 \mathrm{f} 6$

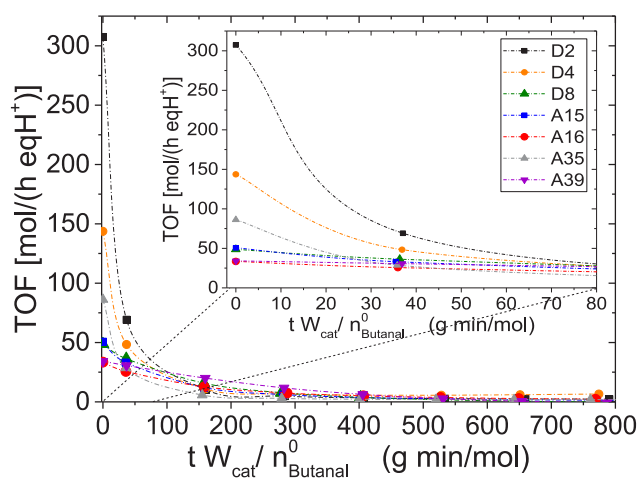

Figure 6. Evolution of turnover frequencies over the resins evaluated vs contact time. $T=50{ }^{\circ} \mathrm{C}, 1.5 \mathrm{MPa}, R_{0}(2 \mathrm{MF} /$ butanal $)=2$, catalyst loading: 1 wt $\%$. The magnification inside highlights the TOF variation at initial contact times.

frequencies with the normalized course of the reaction. As 389 indicative figures, the estimated initial BSB formation rates for 390 the resins D2, D4, D8, A35, A15, A16, and A39 were 1530.8, 391 711.3, 233.7, 458.79, 243.6, 160.4, and $628.74 \mathrm{~mol} /\left(\mathrm{h} \mathrm{kg}_{\text {cat }}\right), 392$ respectively. These values confirm one of the original 393 motivations of the present work, demonstrating that gel-type 394 resins are more efficient catalysts, in terms of yield to target 395 products, product formation rates, and TOF, for reactions 396 involving the formation of water than their macroreticular 397 analogous. Interestingly, the initial TOF for A35 was 398 surprisingly high, even higher than those of D8 and A15 399 resins. An explanation to this fact may arise from the highest 400 acid density $\left(\left[\mathrm{H}^{+}\right] / V_{\mathrm{sp}}\right)$ of this resin that confers a very high 401 initial catalytic activity. However, as the reaction proceeds and 402 products are formed, the diffusional limitations derived from 403 the resistance to swelling play a notable detrimental role in the 404 activity. The immediate aftermath of the oversulfonation, e.g., 405 in A35, is a stiffer structure between polymer chains of the 406 resins because there are more sulfonic groups prone to 407 hydrogen bond the involved chemical species confined in the 408 same space. This leads to reduced accessibility to acid centers 409 and, eventually, to a lower product yield. In this case and as the 410 reaction proceeds, the greater acid capacity of A35 does not 411 make up for its major rigidity, which plays a more prominent 412 role in the catalytic behavior observed.

413

In spite of the similar acid capacity of A16, A15 exhibits a 414 greater surface area $\left(S_{\mathrm{g}}\right)$ in the swollen state (Table 1), which 415 leads to a better macropore diffusion toward the gel phase and 416 an improved initial catalytic activity (Figure 6). On the other 417 hand, A16 has an almost double-fold volume of the gel phase 418 $\left(V_{\text {sp }}\right)$. In other words, a better micropore diffusion of 419 molecules inside the gel phase of A16, yet the access to this 420 gel phase by previous macropore diffusion is more hindered. 421 The balance between these two opposite effects opts slightly 422 for the better permeation, i.e., micropore diffusion, inside the 423 gel phase of A16. As a result, A16 gains activity with the course 424 
Table 2. Compendium of Reported Values of Butanal Conversion and BSB Selectivity and Yield for the HAA of Sylvan with Butanal over Different Catalysts

\begin{tabular}{|c|c|c|c|c|c|c|c|c|c|}
\hline entry & catalyst & $T\left({ }^{\circ} \mathrm{C}\right)$ & $\begin{array}{c}R_{0}(2 \mathrm{MF} / \\
\text { butanal })\end{array}$ & $\begin{array}{l}W_{\text {cat }} \\
(\%)\end{array}$ & $t(\mathrm{~h})$ & $\begin{array}{c}\mathrm{X}_{\text {butanal }} \\
(\%)\end{array}$ & $\begin{array}{c}Y_{\text {butanal }}^{\mathrm{BSB}} \\
(\%)\end{array}$ & $\begin{array}{c}S_{\text {butanal }}^{\text {BSB }} \\
(\%)\end{array}$ & ref \\
\hline 1 & $\mathrm{NbOPO}_{4}$ & 80 & 2.05 & 4 & 5 & 95.3 & 89.5 & & 18 \\
\hline 2 & IGO & 60 & 2 & 2.9 & 6 & & 83 & & 19 \\
\hline 3 & copper triflate & room & 2 & $10^{a}$ & 8 & & 58 & & 20 \\
\hline 4 & Sn- $\beta(12.5)$ zeolite & 100 & 2 & 2 & 10 & & 81 & & 21 \\
\hline 5 & Nafion 212 & 50 & 2 & 3 & 2 & $96.7^{b}$ & $88.4^{b}$ & & 22 \\
\hline 6 & Nafion 212 & 50 & 2 & 3 & 4 & 91 & 81 & 89 & 15 \\
\hline 7 & $\begin{array}{l}\text { 60LS4OPS } 35 \mathrm{OH}^{+} \mathrm{Na} \text {-lignosulfonate derived acidic } \\
\text { carbocatalyst }\end{array}$ & 60 & 2 & 3 & 2 & 99 & 96 & & 24 \\
\hline 8 & $\mathrm{KCC}-1 \mathrm{APSO}_{3} \mathrm{H}$ & 50 & 2 & 3 & 4 & 100 & 94 & 94 & 15 \\
\hline 9 & protonated titanate nanotubes & 50 & 2 & 3 & 4 & $70^{b}$ & $68^{b}$ & & 23 \\
\hline 10 & A15 & 50 & 2 & 3 & 4 & 64 & 47 & 73 & 15 \\
\hline 11 & A15 & 50 & 2 & 3 & 2 & $80^{b}$ & $72^{b}$ & & 22 \\
\hline 12 & A15 & 50 & 2 & 2.3 & 22 & 72 & 59 & 82 & 12 \\
\hline 13 & A15 & 50 & 3 & 1.7 & 22 & 80 & 69 & 86 & 12 \\
\hline 14 & A15 & 50 & 2 & 1.2 & 8 & & $90^{c}$ & & 8 \\
\hline 15 & A15 & 50 & 2 & 1 & 5.4 & 51 & 51 & 100 & this work \\
\hline 16 & A36 & 50 & 2 & 3 & 2 & $72^{b}$ & $70^{b}$ & & 22 \\
\hline 17 & A36 & 50 & 2 & 3 & 4 & 66 & 50 & 75 & 15 \\
\hline 18 & $\mathrm{D} 2$ & 50 & 2 & 1.2 & 8 & & $80^{c}$ & & 8 \\
\hline 19 & D2 & 50 & 2 & 1 & 5.4 & 90 & 90 & 100 & this work \\
\hline
\end{tabular}

${ }^{a}$ Based on mol \%. ${ }^{b}$ Values referred to $2 \mathrm{MF}$ conversion, and therefore, selectivity and yield values are referred to the production of BSB from $2 \mathrm{MF}$. ${ }^{c}$ Yield of $2,2^{\prime}$-butylidenebis[5-methylfuran] with a purity of at least $93 \%$ after $8 \mathrm{~h}$ reaction time.

425 of the reaction, reaching final BSB yields comparable to those 426 obtained with A15 that exhibited higher initial activity.

427 The resin A39 has a similar acid capacity to those of A15 428 and A16, but it is the resin with the lowest cross-linking degree 429 and, therefore, the more flexible structure among the 430 macroreticular resins studied. In the swollen state, A39 exhibits 431 comparable surface area to that of A16 and smaller than A15. 432 However, the swollen state pore volume of A39 significantly 433 exceeds those of A15 and A16, owing to its lower cross-linking 434 degree. As a result, its initial level of activity is comparable to 435 that of the gel-type resin D4 (Figure 6). As the reaction 436 medium becomes more polar by the formation of water, this is 437 translated into an enhanced catalytic activity that increases 438 with the course of the reaction. This can also be clearly seen in 439 the steeper slope between 0 and $400(\mathrm{~g} \mathrm{~min}) / \mathrm{mol}$ in Figure 4, 440 which eventually leads to the high BSB yield reported for this 441 resin at the end of the runs.

442 Aiming to provide a general overview of the current state of 443 the art framework exposed in the introduction and the results 444 reported in this work, Table 2 gathers a collection of butanal 445 conversion, BSB selectivity, and reported yield values for 446 comparison. In some cases, the butanal conversion and its yield 447 toward BSB are equivalent because neither the literature data 448 nor this work detected the presence of butanal-derived 449 byproducts. The HAA of sylvan with butanal over $\mathrm{NbOPO}_{4}$ 450 at $80{ }^{\circ} \mathrm{C}$ resulted in $95.3 \%$ of butanal conversion and $89.5 \%$ of $451 \mathrm{BSB}$ yield after $5 \mathrm{~h} .{ }^{18}$ The BSB yield of $83 \%$ was obtained at 60 $452{ }^{\circ} \mathrm{C}$ using a molar ratio of sylvan to butanal $R_{0}(2 \mathrm{MF} /$ butanal $)$ $453=2$ after $6 \mathrm{~h}$ of reaction over improved graphene oxide $(2.9 \mathrm{wt}$ $454 \%$ loading). ${ }^{19}$ Using copper(II) triflate under solvent-free 455 conditions, $^{20}$ a $58 \%$ BSB yield was obtained after $8 \mathrm{~h}$ of 456 reaction at room temperature, and the catalyst withstood four 457 successive cycles without significant deactivation. In another 458 approach, ${ }^{21}$ for the alkylation step at $100{ }^{\circ} \mathrm{C}$ with a $2 \mathrm{MF} /$ 459 butanal molar ratio of 2 and a catalyst loading of 2 wt \%, Sn460 beta (12.5) zeolite exhibited the best catalytic performance, yielding $81 \%$ of the corresponding alkylated product after $10 \mathrm{~h} .461$ The catalyst was fully recyclable in an aqueous solution with 462 constant product selectivity $(70-72 \%)$ after six successive 463 runs. However, both $2 \mathrm{MF}$ conversion and the corresponding 464 product yield decreased slightly in the last cycle, which was 465 ascribed to the deposition of organic species into zeolite pores. 466

$\mathrm{Li}$ et al. $(2013)^{22}$ reported high $2 \mathrm{MF}$ conversion (96.7\%) 467 and BSB selectivity $(88.4 \%)$ at $50{ }^{\circ} \mathrm{C}$ using a $2 \mathrm{MF} /$ butanal 468 molar ratio of 2 and a catalyst (Nafion 212) loading of 3 wt \% 469 after $2 \mathrm{~h}$. In that study, 2MF conversion for Amberlyst15 470 (A15) and Amberlyst36 (A36) were 81\% and $72 \%, 471$ respectively, with high BSB selectivities (>90\%) without 472 evidence of $2 \mathrm{MF}$ trimer formation. It was concluded that 473 Nafion and Amberlyst sulfonic groups can catalyze the 474 reaction, while H-ZSM-5, H-USY, and H- $\beta$ zeolites are 475 practically inactive due to their smaller pore diameter by 476 considering the size of the BSB molecule. The results were 477 consistent with those reported by Wen et al. (2014), ${ }^{3}$ revealing 478 that zeolite type catalysts are mainly suitable candidates for the 479 HDO step, which must be performed at considerably higher 480 temperatures $\left(200-400{ }^{\circ} \mathrm{C}\right)$. In terms of stability, Nafion 212481 and A15 were remarkably stable for the HAA of $2 \mathrm{MF}$ with 482 butanal after 5 cycles, but A36 exhibited slight deactivation. ${ }^{22} 483$ In addition, the activity of the catalysts tested was consistent 484 with their acid strength. In a different attempt at $50{ }^{\circ} \mathrm{C}$ using 485 the same molar ratio and a $0.15 \mathrm{~g}$ of the protonated titanate 486 nanotube as the catalyst, ${ }^{23}$ high $2 \mathrm{MF}$ conversion $(\sim 70 \%)$ and 487 selectivity were reported after $4 \mathrm{~h}$ of reaction. However, the 488 catalysts deactivated slightly after 3 cycles at the same 489 experimental conditions. In another study, $72 \%$ of butanal 490 conversion with $82 \%$ BSB selectivity was obtained over A15 491 (2.5 wt \%) at $50{ }^{\circ} \mathrm{C}$ using a molar ratio of $2 \mathrm{MF} /$ butanal of 2492 after $22 \mathrm{~h}^{8}$ At identical reaction conditions but a lower catalyst 493 load $(1.15 \mathrm{wt} \%)$ and shorter reaction time $(8 \mathrm{~h})$, the same 494 authors reported striking BSB yield values of $90 \%$ and $80 \%$ for 495 A15 and Dowex 50Wx2, respectively. ${ }^{12}$ 

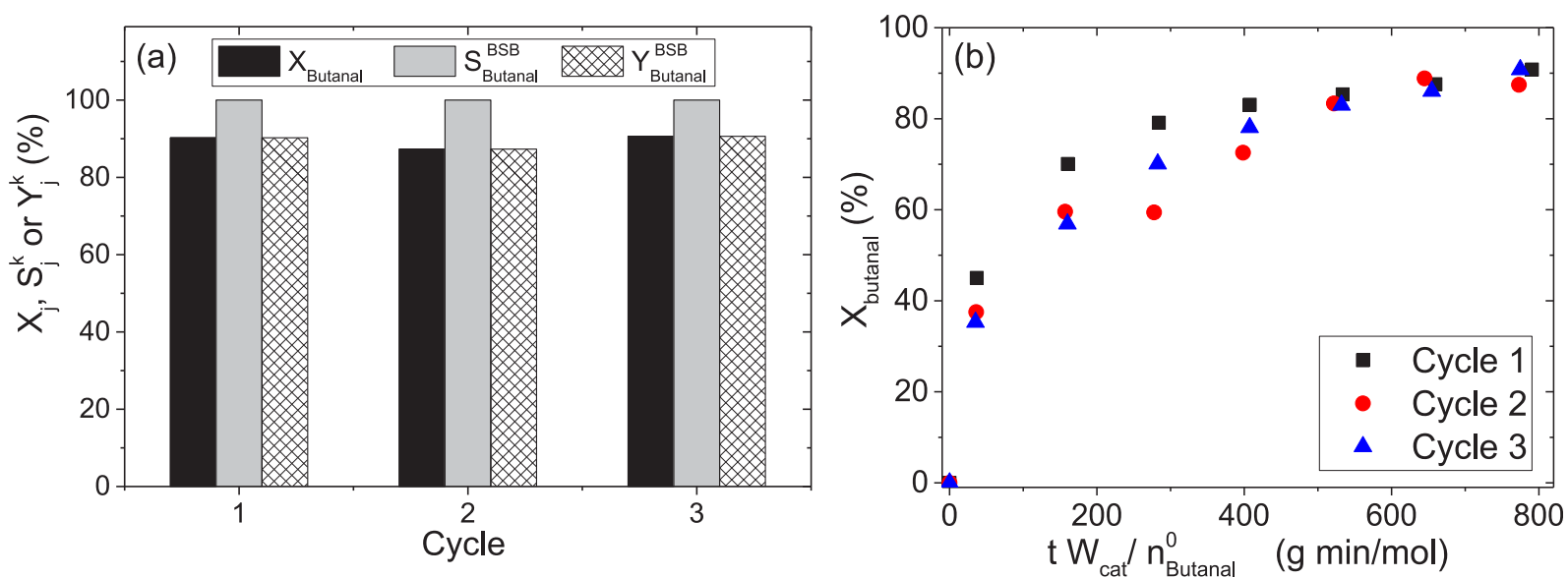

Figure 7. (a) Butanal conversion, selectivity, and yield in each cycle at $t=5.4 \mathrm{~h}$. (b) Evolution of butanal conversion for each cycle. Experimental conditions: $1.5 \mathrm{MPa}, 750 \mathrm{rpm}$, molar ratio $R_{0}(2 \mathrm{MF} /$ butanal $)=2$, catalyst loading 1 wt \% (D2).

497 Using sulfonic acid-based catalysts supported on silica 498 nanoparticles for this reaction at $50{ }^{\circ} \mathrm{C}, R_{0}(2 \mathrm{MF} /$ butanal $)=$ 4992 , catalyst loading of $3 \mathrm{wt} \%$, and reaction time of $4 \mathrm{~h},{ }^{15} \mathrm{KCC}$ $500 \mathrm{APSO}_{3} \mathrm{H}$ catalysts exhibited the highest conversion (100\%) 501 and BSB selectivity (94\%), followed by Nafion 212 (91\% 502 conversion and $89 \%$ selectivity). High butanal conversions of $50364 \%$ and $66 \%$ and selectivities to BSB of $73 \%$ and $75 \%$ were 504 respectively reported over A15 and A36. Na-lignosulfonate 505 (LS)-derived meso/macroporous solid sulfonic carbocatalysts 506 in the solvent-free HAA of $2 \mathrm{MF}$ with butanal, $50760 \mathrm{LS} 40 \mathrm{PS} 350 \mathrm{H}^{+}$exhibited an outstanding carbonyl conversion 508 of $99 \%$ and BSB yield of $96 \%$ along with minimal deactivation 509 (only $2 \%$ in terms of conversion) after three reaction cycles.

510 The comparison between the literature values in Table 2 is 511 not always straightforward because of the different exper512 imental conditions, e.g., catalyst load, reaction temperature, 513 reaction times, or the basis of reported values. However, the 514 butanal conversion follows the expected trend: it increases with 515 an increasing molar ratio of $2 \mathrm{MF}$ to butanal, catalyst load, and 516 reaction time. In general, our reported values for A-15 compare 517 acceptably well with those obtained by Gebresillase et al. ${ }^{15}$ and 518 Corma et al. ${ }^{12}$ under similar conditions. However, a significant 519 discrepancy is observed in comparison to Table 2, entry 14, 520 which can be explained by the longer reaction time of $8 \mathrm{~h}$ used 521 in that study. Interestingly, the studies using catalyst loads 522 above 1.2 wt \% report butanal selectivity to BSB values lower 523 than $100 \%$ irrespectively of the $2 \mathrm{MF}$ /Butanal molar ratio used. 524 Noteworthy, our experiments with 1 wt \% of A-15 were 525 replicated, and butanal-derived byproducts were never 526 detected at $50{ }^{\circ} \mathrm{C}$. This highlights the importance of an 527 optimum catalyst load to avoid side reactions. Conversely, 528 butanal conversion values for D2 resin are more comparable 529 because of more similar experimental conditions. Our values 530 are reasonably similar to those reported by Corma et al. ${ }^{8}$ 531 However, a more rigorous comparison would require to 532 consider the catalyst particle size used in the reference 533 experiment (Table 2, entry 18) since commercial D2 can be 534 supplied in different mesh sizes (50-100, 100-200, and 200$535400)$. An overall outcome emerging from the comparison in 536 Table 2 is that D2 presents a catalytic activity comparable to 537 that reported for the best catalysts previously studied 538 (60LS4OPS35 $\mathrm{OH}^{+} \mathrm{Na}^{-}$-lignosulfonate-derived acidic carboca539 talyst, $\mathrm{KCC}-1 \mathrm{APSO}_{3} \mathrm{H}, \mathrm{NbOPO}_{4}$, and Nafion 212), taking into 540 account the different catalyst loads and reaction times reported. This fact, along with the appealing features of ion- 541 exchange resins in terms of cost, pinpoints D2 as a potential 542 catalyst for the production of BSB by the HAA of $2 \mathrm{MF}$ with 543 butanal.

544

3.2. Effect of Temperature. As mentioned, no significant 545 byproducts derived from sylvan were detected at $50{ }^{\circ} \mathrm{C}$, molar 546 $R_{0}(2 \mathrm{MF} /$ butanal $)=2,1$ wt $\%$ catalyst loading of $\mathrm{D} 2$, and $t=547$ $5.4 \mathrm{~h}$. However, at $60{ }^{\circ} \mathrm{C}$, byproducts began to appear $(3 \% 548$ GC), representing their presence of $10 \% \mathrm{GC}$ at $70{ }^{\circ} \mathrm{C}$ and $23 \% 549$ $\mathrm{GC}$ at $90{ }^{\circ} \mathrm{C}$. In agreement with the literature, $, 14,15,21$ the 550 main byproduct detected, of higher molar mass than BSB, was 551 the 2MF trimer (5,5-bisylvyl-2-pentanone). Unfortunately, the 552 rest of the byproducts could not be properly identified by MS 553 due to their low amount. The reaction pathway for the 554 generation of BSB and oligomers of sylvan is illustrated in 555 Schemes 1 and 2, respectively. Even though both butanal and 556 $2 \mathrm{MF}$ conversion increase with temperature, a significant 557 decrease in the $2 \mathrm{MF}$ selectivity to BSB eventually leads to an 558 important depletion of the BSB yield. Thus, it can be 559 concluded that, to minimize byproducts formation, temper- 560 atures below $60{ }^{\circ} \mathrm{C}, R_{0}(2 \mathrm{MF} /$ butanal $)=2$, and 1 wt $\%$ of 561 catalyst load are the most appropriate conditions for 562 conducting the HAA reaction between sylvan and butanal 563 catalyzed by the acidic ion-exchange resin D2.

3.3. Reusability. As the most promising catalyst, D2 was 565 reused three times by following the previously detailed 566 reactivation procedure. Figure $7 \mathrm{a}$ shows conversion, selectivity, $567 \mathrm{f7}$ and yield obtained at the end of the runs in each cycle. The 568 butanal selectivity to BSB (100\%) remained unchanged after 569 three cycles. Butanal conversion, however, decreased slightly 570 (less than 3\%), which can be considered negligible, taking into 571 account the experimental uncertainty. Under similar exper- 572 imental conditions, $^{22}$ no deactivation was detected over A15 573 after five cycles, basing the calculations on the $2 \mathrm{MF}$ conversion 574 and yield as a reference. For a better assessment of the catalyst 575 stability, Figure $7 \mathrm{~b}$ depicts the evolution of butanal conversion 576 in each cycle. Noteworthy, some signs of loss of initial catalytic 577 activity $(\sim 10 \%)$ were observed at the beginning of the runs, 578 yet identical conversion values were reported for contact times 579 of $500(\mathrm{~g} \mathrm{~min}) / \mathrm{mol}$ onward. Although the performance of the 580 most promising catalyst evaluated in this work must still be 581 evaluated in different practical scenarios, particularly in flow 582 conditions with long times on stream for its industrial 583 application, the results herein reported reveal a positive and 584 
585 favorable insight for considering D2 resin as a potential catalyst 586 with high activity, selectivity, and stability, for the HAA 587 reaction of $2 \mathrm{MF}$ with butanal.

\section{CONCLUSIONS}

588 The catalytic hydroxyalkylation/alkylation of 2-methylfuran 589 with butanal at a stoichiometric molar ratio can be successfully 590 catalyzed by ion-exchange resins at the temperature range 50$59190{ }^{\circ} \mathrm{C}$. Butanal conversion increases with temperature but so 592 does the formation of 2-methylfuran oligomers, leading to an 593 overall decrease of yield to BSB. The catalytic activity and yield 594 to the target product of gel-type resins are superior to those of 595 macroreticular ones due to their ability to swell during the 596 reaction by the formation of water, which promotes enhanced 597 accessibility to active sites. Among the resins evaluated, the 598 most promising catalyst (Dowex 50Wx2) presents the lowest 599 cross-linking degree and achieves notably high butanal 600 conversion (90\%) and selectivity to BSB (100\%) at $50{ }^{\circ} \mathrm{C}$, 601 without significant formation of 2 -methylfuran oligomers. 602 Macroreticular resins with low content of a cross-linking 603 agent also give acceptable catalytic behavior. The catalytic 604 activity rank observed in terms of final yield to target product 605 can be rationalized on the basis of the resin morphological 606 properties: the activity increase with decreasing acid density, 607 i.e., the ratio of acid capacity to the volume of the swollen 608 polymer. This fact highlights the paramount importance of 609 enhanced accessibility to improve diffusivity for the present 610 reaction system that involves products of significant molecular 611 volume. The reusability of the most prominent catalyst (D2) 612 has been evaluated after three reaction cycles, and the results 613 suggest that it is a stable catalyst with the industrial prospective 614 application for the studied reaction system.

\section{AUTHOR INFORMATION}

\section{Corresponding Author}

617 Eliana Ramírez - Department of Chemical Engineering and

618 Analytical Chemistry, Faculty of Chemistry, University of

619 Barcelona, 08028 Barcelona, Spain; orcid.org/0000-

620 0001-8695-1533; Email: eliana.ramirez-rangel@ub.edu

\section{Authors}

622 Rodrigo Soto - Synthesis and Solid State Pharmaceutical 623 Centre (SSPC), Bernal Institute, Department of Chemical 624 and Environmental Science, University of Limerick, Limerick 625 V94 T9PX, Ireland; 이잉.org/0000-0002-9988-7494

626 Roger Bringué - Department of Chemical Engineering and 627 Analytical Chemistry, Faculty of Chemistry, University of 628 Barcelona, 08028 Barcelona, Spain

629 Montserrat Iborra - Department of Chemical Engineering

630 and Analytical Chemistry, Faculty of Chemistry, University of

631 Barcelona, 08028 Barcelona, Spain

632 Javier Tejero - Department of Chemical Engineering and 633 Analytical Chemistry, Faculty of Chemistry, University of 634 Barcelona, 08028 Barcelona, Spain; 이잉.org/0000$635 \quad 0002-2708-5273$

636 Complete contact information is available at: 637 https://pubs.acs.org/10.1021/acs.iecr.0c04308

\section{Notes}

639 The authors declare no competing financial interest.

\section{ACKNOWLEDGMENTS}

640

The authors are grateful to MINECO (CTQ2014-56618-R 641 Grant) for their financial support and Rohm and Haas France 642 S.A.S for providing ion-exchange resins samples. Also, we 643 thank Laura González Saladich for the help with the graphic 644 design of the artwork.

645

\section{NOTATION}

$\mathrm{ABE}=$ acetone-butanol-ethanol

A15 = Amberlyst 15

A16 $=$ Amberlyst16

A35 = Amberlyst35

A39 = Amberlyst39

$\mathrm{BSB}=1,1$-bysylvylbutane

$\mathrm{DVB}=$ divinyl benzene

$\mathrm{D} 2$ = Dowex 50Wx2

$\mathrm{D} 4=$ Dowex $50 \mathrm{Wx} 4$

D8 $=$ Dowex 50Wx8

$\mathrm{GC}=$ gas chromatography

HAA = hydroxyalkylation/alkylation

648

649

650

651

652

653

654

655

656

$\mathrm{HDO}=$ hydrodeoxygenation

$\left[\mathrm{H}^{+}\right]=$acid capacity, $\mathrm{mmol} / \mathrm{g}$

$\left[\mathrm{H}^{+}\right] / V_{\mathrm{sp}}=$ acid site density in swollen resins, $\mathrm{mmol} / \mathrm{cm}^{3} \quad 661$

IGO = improved graphene oxide

$(\mathrm{LS})=$ Na-lignosulfonate

$n_{\text {butanal }}^{0}=$ initial mole of butanal

$n_{\text {butanal }}=$ mole of butanal

$n_{\mathrm{BSB}}=$ mole of BSB

PS-DVB = polystyrene-divinylbenzene-based resins

$R_{0(2 \mathrm{MF} / \text { butanal })}=$ molar ratio of sylvan to butanal

$S^{\mathrm{BSB}}$ butanal = selectivity of butanal toward BSB

$S_{\mathrm{g}}=$ specific area, $\mathrm{m}^{2} / \mathrm{g}$

$t=$ time, $\min$

$T=$ temperature, ${ }^{\circ} \mathrm{C}$

TCD $=$ thermal conductivity detector

$T_{\max }=$ maximum temperature of resins for stability, ${ }^{\circ} \mathrm{C}$

$V_{\mathrm{g}}=$ specific volume of pores, $\mathrm{cm}^{3} / \mathrm{g}$

$V_{\text {sp }}=$ swollen specific volume of gel phase, $\mathrm{cm}^{3} / \mathrm{g}$

$W_{\text {cat }}=$ dried mass of catalyst, $\mathrm{g}$

$\mathrm{X}_{\text {butanal }}=$ butanal conversion

$\mathrm{Y}^{\mathrm{BSB}}{ }_{\text {butanal }}=$ yield of butanal toward BSB

$2 \mathrm{MF}=2$-methylfuran

657

658

659

662

663

664

665

666

667

668

669

670

671

672

673

674

675

676

677

678

679

680

\section{REFERENCES}

(1) Garcia-Ortiz, A.; Arias, K. S.; Climent, M. J.; Corma, A.; Iborra, 682 S. Transforming methyl levulinate into biosurfactants and bio- 683 lubricants by chemoselective reductive etherification with fatty 684 alcohols. ChemSusChem 2020, 13, 707-714.

(2) Climent, M. J.; Corma, A.; Iborra, S. Conversion of biomass 686 platform molecules into fuel additives and liquid hydrocarbon fuels. 687 Green Chem. 2014, 16, 516-547.

(3) Wen, C.; Barrow, E.; Hattrick-Simpers, J.; Lauterbach, J. One- 689 step production of long-chain hydrocarbons from waste-biomass- 690 derived chemicals using bi-functional heterogeneous catalysts. Phys. 691 Chem. Chem. Phys. 2014, 16, 3047-3054.

(4) Zainol, M. M.; Amin, N. A. S.; Asmadi, M.; Ramli, N. A. S. 693 Esterification of levulinic acid to ethyl levulinate using liquefied oil 694 palm frond-based carbon cryogel catalyst. BioEnergy Res. 2019, 12, 695 359-369.

(5) Hamdi, J.; Diehl, B. N.; Kilgore, K.; Lomenzo, S. A.; Trudell, M. 697 L. Halloysite-catalyzed esterification of bio-Mass derived acids. ACS 698 Omega 2019, 4, 19437-19441.

699

(6) Kumar, P.; Varkolu, M.; Mailaram, S.; Kunamalla, A.; Maity, S. 700

K. Biorefinery polyutilization systems: Production of green trans- 701 
702 portation fuels from biomass. In Polygeneration with Polystorage Chem. 703 Energy Hubs; Khalilpour, K. R., Ed.; Elsevier: London, 2018; pp 373704407.

705 (7) Ramírez, E.; Bringué, R.; Fité, C.; Iborra, M.; Tejero, J.; Cunill, $706 \mathrm{~F}$. Role of ion-exchange resins as catalyst in the reaction-network of 707 transformation of biomass into biofuels. J. Chem. Technol. Biotechnol. 708 2017, 92, 2775-2786.

709 (8) Corma, A.; De La Torre, O.; Renz, M. Production of high quality 710 diesel from cellulose and hemicellulose by the Sylvan process: 711 Catalysts and process variables. Energy Environ. Sci. 2012, 5, 63287126344.

713 (9) Gandarias, I.; García-Fernández, S.; Obregón, I.; Agirrezabal714 Telleria, I.; Arias, P. L. Production of 2-methylfuran from biomass 715 through an integrated biorefinery approach. Fuel Process. Technol. 716 2018, 178, 336-343.

717 (10) Wang, C.; Xu, H.; Daniel, R.; Ghafourian, A.; Herreros, J. M.; 718 Shuai, S.; Ma, X. Combustion characteristics and emissions of 2719 methylfuran compared to 2,5-dimethylfuran, gasoline and ethanol in a 720 DISI engine. Fuel 2013, 103, 200-211.

721 (11) Zhang, X.; Deng, Q.; Han, P.; Xu, J.; Pan, L.; Wang, L.; Zou, J.$722 \mathrm{~J}$. Hydrophobic mesoporous acidic resin for hydroxyalkylation/ 723 alkylation of 2-methylfuran and ketone to high-density biofuel. 724 AIChE J. 2017, 63, 680-688.

725 (12) Corma, A.; de la Torre, O.; Renz, M.; Villandier, N. Production 726 of high-quality diesel from biomass waste products. Angew. Chem., Int. 727 Ed. 2011, 50, 2375-2378.

728 (13) Schlögl, R. Chemical Energy Storage; De Gruyter: Berlin, 2013. 729 (14) Green, S. K. Production of Renewable Fuels and Chemicals from 730 Biomass-Derived Furan Compounds. Ph.D.Thesis, University of 731 Massachusetts, November 2014.

732 (15) Gebresillase, M. N.; Shavi, R.; Seo, J. G. A comprehensive 733 investigation of the condensation of furanic platform molecules to 734 C14-C15 fuel precursors over sulfonic acid functionalized silica 735 supports. Green Chem. 2018, 20, 5133-5146.

736 (16) Ishigaki, A.; Shono, T. The cationic oligomerization of 2737 Methylfuran and the characteristics of the oligomers. Bull. Chem. Soc. 738 Jpn. 1974, 47, 1467-1470.

739 (17) Nakagawa, Y.; Tamura, M.; Tomishige, K. Recent development 740 of production technology of diesel- and jet-fuel-range hydrocarbons 741 from inedible biomass. Fuel Process. Technol. 2019, 193, 404-422.

742 (18) Xia, Q.; Xia, Y.; Xi, J.; Liu, X.; Zhang, Y.; Guo, Y.; Wang, Y. 743 Selective One-Pot Production of high-grade diesel-range alkanes from 744 furfural and 2-Methylfuran over $\mathrm{Pd} / \mathrm{NbOPO}_{4}$. ChemSusChem 2017, 745 10, 747-753.

746 (19) Dutta, S.; Bohre, A.; Zheng, W.; Jenness, G. R.; Nunez, M.; 747 Saha, B.; Vlachos, D. G. Solventless C-C coupling of low carbon 748 furanics to high carbon fuel precursors using an improved graphene 749 oxide carbocatalyst. ACS Catal. 2017, 7, 3905-3915.

750 (20) Muthyala, M. K.; Rao, V. K.; Kumar, A. Cu(OTf) $)_{2}$ Catalyzed 751 synthesis of bis(5-methyl-2-furyl)methanes by condensation of 2752 Methylfuran with carbonyl compounds under solvent free conditions. 753 Chin. J. Chem. 2011, 29, 1483-1488.

754 (21) Li, H.; Gui, Z.; Yang, S.; Qi, Z.; Saravanamurugan, S.; Riisager, 755 A. Catalytic tandem reaction for the production of jet and diesel fuel 756 range alkanes. Energy Technol. 2018, 6, 1060-1066.

757 (22) Li, G.; Li, N.; Yang, J.; Wang, A.; Wang, X.; Cong, Y.; Zhang, T. 758 Synthesis of renewable diesel with the 2-methylfuran, butanal and 759 acetone derived from lignocellulose. Bioresour. Technol. 2013, 134, $76066-72$.

761 (23) Li, S.; Li, N.; Li, G.; Li, L.; Wang, A.; Cong, Y.; Wang, X.; Xu, 762 G.; Zhang, T. Protonated titanate nanotubes as a highly active catalyst 763 for the synthesis of renewable diesel and jet fuel range alkanes. Appl. 764 Catal., B 2015, 170-171, 124-134.

765 (24) Konwar, L.; J. Samikannu, A.; Mäki-Arvela, P.; Mikkola, J. P. 766 Efficient C-C coupling of bio-based furanics and carbonyl compounds 767 to liquid hydrocarbon precursors over lignosulfonate derived acidic 768 carbocatalysts. Catal. Sci. Technol. 2018, 8, 2449-2459.

769 (25) Lachter, E. R.; Rodrigues, J. A.; Teixeira, V. G.; Mendonca, R. 770 H.; Ribeiro, P. S.; Villabona-Estupinan, S. Use of ion-exchange resins in alkylation reactions. Applications of Ion Exchange Materials in 771 Chemical and Food Industries 2019, 35-74.

(26) Guilera, J.; Bringué, R.; Ramírez, E.; Iborra, M.; Tejero, J. 773 Synthesis of ethyl octyl ether from diethyl carbonate and 1-octanol 774 over solid catalysts. A screening study. Appl. Catal., A 2012, 413-414, 775 21-29.

(27) Corain, B.; Zecca, M.; Jeřábek, K. Catalysis and polymer 777 networks - the role of morphology and molecular accessibility. J. Mol. 778 Catal. A: Chem. 2001, 177, 3-20.

(28) Tejero, M.A.; Ramirez, E.; Fite, C.; Tejero, J.; Cunill, F. 780 Esterification of levulinic acid with butanol over ion Exchange resins. 781 Appl. Catal., A 2016, 517, 56-66.

(29) Albright, R. L. Porous polymers as an anchor for catalysis. 783 React. Polym., Ion Exch., Sorbents 1986, 4, 155-174. 784

(30) Guyot, A. Synthesis and structure of polymer supports. In 785 Synthesis and Separations using polymer supports; Sherrington, D. C., 786 Hodge, P., Eds.; Wiley: Chichester, 1988; pp 15.

(31) Sterchele, S.; Centomo, P.; Zecca, M.; Hanková, L.; Jeřábek, K. 788 Dry- and swollen-state morphology of novel high surface area 789 polymers. Microporous Mesoporous Mater. 2014, 185, 26-29. 790

(32) Jeřábek, K. Inverse steric exclusion chromatography as a tool 791 for morphology characterization. In Strategies in size exclusion 792 chromatography; Potschka, M., Dubin, P. L., Eds.; American Chemical 793 Society Publications: WA, 1996; pp 211-224.

(33) Jeřábek, K. Determination of pore volume distribution from 795 size exclusion chromatography data. Anal. Chem. 1985, 57, 1595- 796 1597.

(34) Ogston, A. G. The spaces in a uniform random suspension of 798 fibres. Trans. Faraday Soc. 1958, 54, 1754-1757. 